Original Article

\title{
The Digestive Tract Morphology and Clark Index of Mud Sleeper Butis koilomatodon Living in some Coastal and Estuarine Areas Belonging to Tra Vinh, Soc Trang, Bac Lieu and $\mathrm{Ca} \mathrm{Mau}$
}

\author{
Dinh Minh Quang ${ }^{1, *}$, Nguyen Thi Nha $Y^{1}$, Lam Thi Huyen Tran ${ }^{1,2}$, Phan Thanh Gieo ${ }^{1,3}$ \\ ${ }^{1}$ Can Tho University, Campus II, 3/2 street, Xuan Khanh, Ninh Kieu,Can Tho, Vietnam \\ ${ }^{2}$ University of Cuu Long, Phu Quoi, Vinh Long, Vietnam \\ ${ }^{3}$ Kien Giang University, 320A, AH 61, Vinh Hoa Hiep, Chau Thanh, Kien Giang, Vietnam
}

Received 06 April 2020

Revised 07 August 2020; Accepted 17 August 2020

\begin{abstract}
This study provides new data on the characteristics of the gastrointestinal morphology and the variation of Clark index according to sexes, fish size groups, seasons and sampling sites of mud sleeper Butis koilomatodon, one of the commercially valuable gobiid species in the Mekong Delta. Analytical results of 1,227 fish samples collected monthly from April 2019 to March 2020 in Duyen Hai (Tra Vinh), Cu Lao Dung (Soc Trang), Tran De (Soc Trang), Hoa Binh (Bac Lieu), Dong Hai (Bac Lieu) and Dam Doi (Ca Mau) showed that this species had a slightly sloping mouth, the length between two jaws was relatively equal, teeth were small and grew in long lines on both jaws with the outer row having larger teeth. The length of the gut was shorter than the total length of the fish and so this species belonged to predator group. The Clark index depended on length of fish, sampling time and site but excluding season and gender. It was also effected by interactions of fish length $\mathrm{x}$ season; fish length $\mathrm{x}$ site and season $\mathrm{x}$ site. Conversly, the interactions of gender $\mathrm{x}$ season and gender $\mathrm{x}$ site did not impact on the Clark index. The outcomes of research will provide useful data in artificial aquaculture Butis koilomatodon.
\end{abstract}

Keywords: Butis koilomatodon, Clark index, coastal and estuarine area, gastrointestinal.

\footnotetext{
${ }^{*}$ Corresponding author.

Email address: dmquang@ctu.edu.vn

https://doi.org/10.25073/2588-1140/vnunst.5051
} 


\title{
Hình thái ống tiêu hóa và hệ số béo Clark của cá bống lưng cao Butis koilomatodon phân bố ở một số vùng cửa song ven biển thuộc các tỉnh Trà Vinh, Sóc Trăng, Bạc Liêu và Cà Mau
}

\author{
Đinh Minh Quang ${ }^{1, *}$, Nguyễn Thị Nhã Ý ${ }^{1}$, Lâm Thị Huyền Trân ${ }^{1,2}$, \\ Phan Thanh Giẻo ${ }^{1,3}$ \\ ${ }^{1}$ Truoòng Đại học Cần Tho, Khu II, Đường 3/2, Xuân Khánh, Ninh Kiều, Tp. Cần Tho; Việt Nam \\ ${ }^{2}$ Truờng Đại học Cửu Long, Quốc Lộ 1A, Phú Quới, Long Hồ, Vĩnh Long, Việt Nam \\ ${ }^{3}$ Truòng Đại học Kiên Giang, 320A, Quốc Lộ 61, Vĩnh Hoà Hiệp, Châu Thành, Kiên Giang, Việt Nam
}

Nhận ngày 06 tháng 04 năm 2020

Chỉnh sửa ngày 07 tháng 08 năm 2020; Chấp nhận đăng ngày 17 tháng 08 năm 2020

\begin{abstract}
Tóm tắt: Nghiên cứu này cung cấp dẫn liệu mới về đặc điểm hình thái ống tiêu hóa và sự biến động của hệ số béo Clark theo giới tính, nhóm chiều dài cá, mùa thu mẫu và điểm thu mẫu của loài cá bống lưng cao Butis koilomatodon, một trong những loài cá bống có giá trị thương phẩm ở vùng Đồng bằng sông Cửu Long. Kết quả phân tích 1.227 mẫu cá thu được liên tục mỗi tháng một lần, từ tháng 04/2019 đến tháng 03/2020 tại 6 địa điểm tại các huyện Duyên Hải (Trà Vinh), Cù Lao Dung (Sóc Trăng), Trần Đề (Sóc Trăng), Hòa Bình (Bạc Liêu), Đông Hải (Bạc Liêu) và Đầm Dơi (Cà Mau) thấy được loài này có miệng hơi chếch về phía dưới, chiều dài giữa hai hàm tương đối bằng nhau, răng nhỏ và mọc thành hàng dài trên cả hai hàm với hàng ngoài có răng lớn hơn. Chiều dài của ruột cá ngắn hơn chiều dài tổng của cá và loài này thuộc nhóm cá ăn động vật. Giá trị Clark của loài này thay đổi theo chiều dài của cá, thời gian thu mẫu và điểm thu mẫu cũng như sự tác động kết hợp của chiều dài cá $\times$ mùa, chiều dài cá $\times$ điểm thu mẫu, mùa $\times$ điểm thu mẫu, nhưng không chịu ảnh hưởng bởi mùa, giới tính và giới tính $\times$ mùa, giới tính $\times$ điểm thu mẫu. Kết quả này góp phần cung cấp dẫn liệu cho các nghiên cứu nuôi nhân tạo loài cá này trong tương lai.
\end{abstract}

Tư khóa: Butis koilomatodon, hệ số béo Clark, hình thái ống tiêu hóa, vùng cửa sông ven biển.

\section{Mở đầu}

Cá bống lưng cao Butis koilomatodon (Bleeker, 1849) sống tập trung ở cửa sông lớn có chất đáy là bùn cát, một số vùng nước nông và khe đá [1]. Cá bống còn có giá trị dinh dưỡng cao hơn nhiều loài cá có giá trị kinh tế khác ở biển [2]. Hơn nữa, trong nhiều năm gần đây, việc đánh bắt quá mức đã làm cho nguồn lợi cá bống giảm sút nhanh chóng, nhiều loài đã trở nên cạn kiệt. Tuy nhiên, đến nay có rất ít nghiên cứu về đối tượng này, đặc biệt các nghiên cứu về hình thái ngoài của ống tiêu hóa và sự thay đổi của chỉ số Clark với kích thước của cá, địa điểm và mùa vẫn chưa được biết đến. Hình thái ống tiêu hóa được dùng để xác định tính ăn của cá [3]. Chỉ số

\footnotetext{
* Tác giả liên hệ.

Địa chỉ email: dmquang@ctu.edu.vn

https://doi.org/10.25073/2588-1140/vnunst.5051
} 
Clark biểu thị cho khả năng tích lũy năng lượng của cá [4]. Nghiên cứu này nhằm cung cấp dẫn liệu về hình thái ống tiêu hóa và hệ số Clark của cá và sự biến đổi giá trị của hệ số Clark theo giới tính, nhóm chiều dài, mùa, điểm và thời gian thu mẫu. Kết quả của đề tài sẽ là cơ sở hữu ích cho việc nghiên cứu nuôi nhân tạo loài này trong tương lai.

\section{Vật liệu và phương pháp}

\subsection{Thu và phân tích mẫu}

Mẫu cá bống lưng cao Butis koilomatodon được thu dọc theo vùng cửa sông ven biển ở huyện Duyên Hải (Trà Vinh), Cù Lao Dung (Sóc Trăng), Trần Đề (Sóc Trăng), Hòa Bình (Bạc Liêu), Đông Hải (Bạc Liêu) và Đầm Dơi (Cà Mau) (Hình 1) bằng lưới kéo đáy (có kích thước mắt lưới phần đụt là $2 \mathrm{a}=15 \mathrm{~mm}$ ). Mẫu được thu ngẫu nhiên, định kỳ 1 lần/tháng với nhiều kích cỡ cá khác nhau và thu liên tục trong một năm từ tháng 04/2019 đến tháng 03/2020.

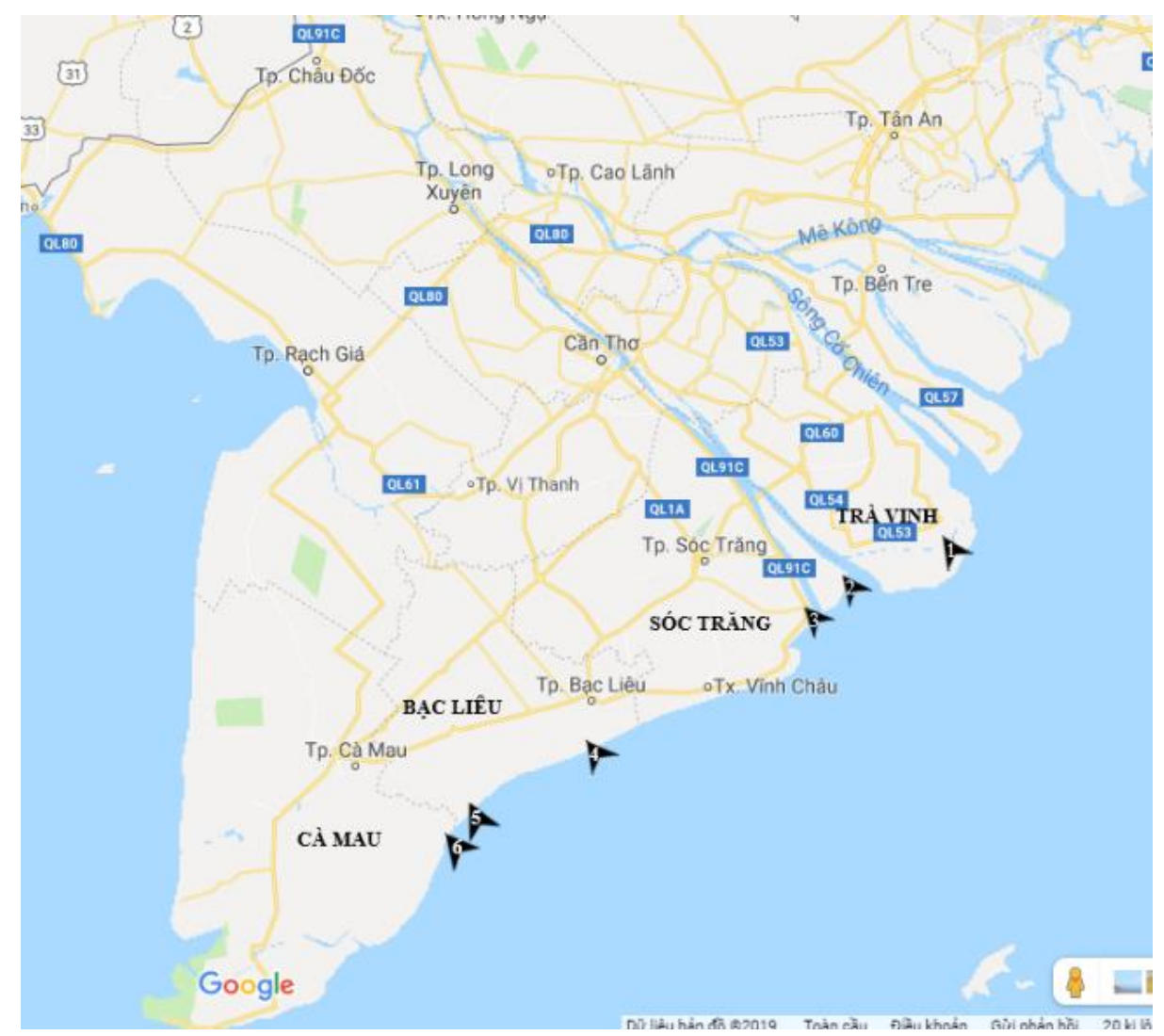

Hình 1. Sơ đồ sáu điểm thu mẫu

[Dấu mũi tên: địa điểm thu mẫu; 1: Duyên Hải (Trà Vinh), 2: Cù Lao Dung (Sóc Trăng), 3: Trần Đề (Sóc Trăng), 4: Hòa Bình (Bạc Liêu), 5: Đông Hải (Bạc Liêu), 6: Đầm Dơi (Cà Mau); Nguồn: Google Map]

Mẫu cá sau khi thu được bảo quản bằng cách ngâm trong formol $10 \%$ (1 formoline: 9 nước) trước khi mang về phòng thí nghiệm. Sau khi mang về Phòng thí nghiệm Động vật, Bộ môn
Sinh học, Khoa Sư phạm, mẫu cá được định loại dựa vào đặc điểm hình thái ngoài đã được mô tả bởi Trần Đắc Định và cộng sự (2013) [5]. Giới tính được xác định dựa vào đặc điểm hình thái 
của gai sinh dục (tam giác nhọn ở cá đực và oval ở cá cái). Mẫu cá được xác định khối lượng (W; chính xác đến $0,01 \mathrm{~g}$ ) và chiều dài (TL; chính xác đến $0,1 \mathrm{~cm}$ ) trước khi giải phẫu lấy ống tiêu hóa.

Hình thái ống tiêu hóa được mô tả dựa trên phương pháp nghiên cứu của Nikolsky (1963) [3]. Chỉ số Clark được tính dựa vào khối lượng cá không nội quan ( $\mathrm{W}_{\mathrm{o}}$; chính xác đến $0,01 \mathrm{~g}$ ) theo công thức:

$$
\text { Clark }=\frac{\mathrm{W}_{\mathrm{o}} \times 100}{\mathrm{TL}^{3}}
$$

Trong đó, $\mathrm{W}_{\mathrm{o}}$ : khối lượng cá không nội quan; TL: tổng chiều dài của cá [4].

\subsection{Xử lý số liệu}

Sự biến động của giá trị Clark theo giới tính, nhóm chiều dài cá và mùa được xác định bằng $\mathrm{t}$ test. Phương pháp phân tích phương sai một nhân tố (1-way ANOVA) được dùng để kiểm tra sự biến động của giá trị Clark theo điểm và tháng thu mẫu. Phương pháp phân tích phương sai hai nhân tố (2-way ANOVA) được dùng để xác định sự ảnh hưởng của giới tính $\times$ mùa, giới tính $\times$ điểm thu mẫu, nhóm chiều dài cá $\times$ mùa, nhóm chiều dài cá $\times$ điểm thu mẫu và mùa $\times$ điểm thu mẫu đến sự thay đổi của giá trị Clark. Kích thước của cá được chia thành 2 nhóm dựa vào chiều dài thành thục đầu tiên ở mỗi địa điểm thu mẫu (chiều dài thành thục đầu tiên: tại Duyên Hải: $6,95 \mathrm{~cm}$; Cù Lao Dung: $8,61 \mathrm{~cm}$; Trần Đề: 6,93 cm; Hòa Bình: 5,50 cm; Đông Hải: 5,09 cm;
Đầm Dơi: $6,95 \mathrm{~cm}$ ), với nhóm 1 có kích thước nhỏ hơn chiều dài thành thục đầu tiên (tài liệu chưa công bố). Phần mềm SPSS v. 21 được dùng để xử lý thống kê. Tất cả phép thử được xác định ở mức ý nghĩa $5 \%$.

\section{Kết quả và thảo luận}

\subsection{Hình thái ống tiêu hóa}

Trong thời gian từ tháng $04 / 2019$ đến tháng 03/2020 đã thu được tổng cộng 1.227 cá thể đực và cái (Bảng 1 ). Kết quả phân tích cho thấy loài cá bống lưng cao Butis koilomatodon có miệng hơi chếch về phía dưới, chiều dài hàm trên và hàm dưới tương đối bằng nhau cho thấy loài này thuộc nhóm cá miệng giữa và thường bắt mồi ở tầng giữa. Răng loài này nhỏ, mọc thành hàng dài trên cả hai hàm của cá, hàng phía ngoài có răng lớn hơn và hơi cong về phía miệng cá (Hình 2). Loài cá này có chiều dài thân từ $2,9 \mathrm{~cm}$ đến $11,1 \mathrm{~cm}(7,16 \pm 0,03 \mathrm{SE} \mathrm{cm})$, chiều dài ruột dao động từ $1,0 \mathrm{~cm}$ đến $8,0 \mathrm{~cm}(3,99 \pm 0,03 \mathrm{SE} \mathrm{cm}$; Hình 3), chỉ số RLG của loài này dao động từ 0,17 dến $1,41(0,56 \pm 0,00 \mathrm{SE})$. Chiều dài của ruột cá ngắn hơn chiều dài tổng của cá chứng tỏ loài cá này thuộc nhóm cá ăn động vật. Kết quả này cũng được tìm thấy ở một số loài cá bống ở khu vực Đồng bằng sông Cửu Long như: Glossogobius giuris [6], Glossogobius sparsipapillus [7], Oxyeleotris urophthalmus [8], Eleotris melanosoma [9,10], Periophthalmodon schlosseri [11].

Bảng 1. Số lượng cá thu được tại sáu điểm thu mẫu ở khu vực nghiên cứu

\begin{tabular}{|c|c|c|c|c|c|c|c|c|c|c|c|c|}
\hline \multirow{2}{*}{ Tháng } & \multicolumn{2}{|c|}{ Duyên Hải } & \multicolumn{2}{c|}{ Cù Lao Dung } & \multicolumn{2}{c|}{ Trần Đề } & \multicolumn{2}{|c|}{ Hòa Bình } & \multicolumn{2}{|c|}{ Đông Hải } & \multicolumn{2}{c|}{ Đầm Dơi } \\
\cline { 2 - 12 } & Đực & Cái & Đực & Cái & Đực & Cái & Đực & Cái & Đực & Cái & Đực & Cái \\
\hline $04 / 2019$ & 6 & 2 & 3 & 1 & 12 & 3 & 7 & 25 & 17 & 4 & 21 & 6 \\
\hline $05 / 2019$ & 10 & 1 & 10 & 2 & 8 & & 22 & 9 & 12 & 11 & 19 & 9 \\
\hline $06 / 2019$ & 8 & 5 & 6 & 4 & 8 & & 25 & 5 & 14 & 3 & 15 & 14 \\
\hline $07 / 2019$ & 8 & & 3 & 1 & 9 & 3 & 24 & 6 & 19 & 0 & 16 & 4 \\
\hline $08 / 2019$ & 7 & 5 & 6 & 1 & 9 & 1 & 24 & 6 & 12 & 8 & 11 & 10 \\
\hline $09 / 2019$ & 9 & 1 & 5 & 4 & 9 & 3 & 24 & 6 & 11 & 5 & 14 & \\
\hline $10 / 2019$ & 12 & & 5 & 1 & 7 & 6 & 21 & 7 & 14 & 4 & 19 & 2 \\
\hline $11 / 2019$ & 8 & 2 & 3 & 1 & 15 & & 21 & 9 & 16 & 1 & 7 & 9 \\
\hline $12 / 2019$ & 9 & 3 & 1 & 4 & 7 & 2 & 23 & 8 & 1 & 13 & 11 & 13 \\
\hline $01 / 2020$ & 19 & 5 & 3 & 2 & 4 & 1 & 28 & 2 & 15 & 2 & 15 & 15 \\
\hline $02 / 2020$ & 9 & 11 & 5 & 1 & 2 & 1 & 16 & 7 & 14 & 4 & 20 & 10 \\
\hline $03 / 2020$ & 26 & 4 & 12 & 2 & 3 & 1 & 20 & 6 & 13 & 3 & 24 & 6 \\
\hline Tồng & 131 & 39 & 162 & 24 & 93 & 21 & 255 & 96 & 158 & 58 & 192 & 98 \\
\hline
\end{tabular}



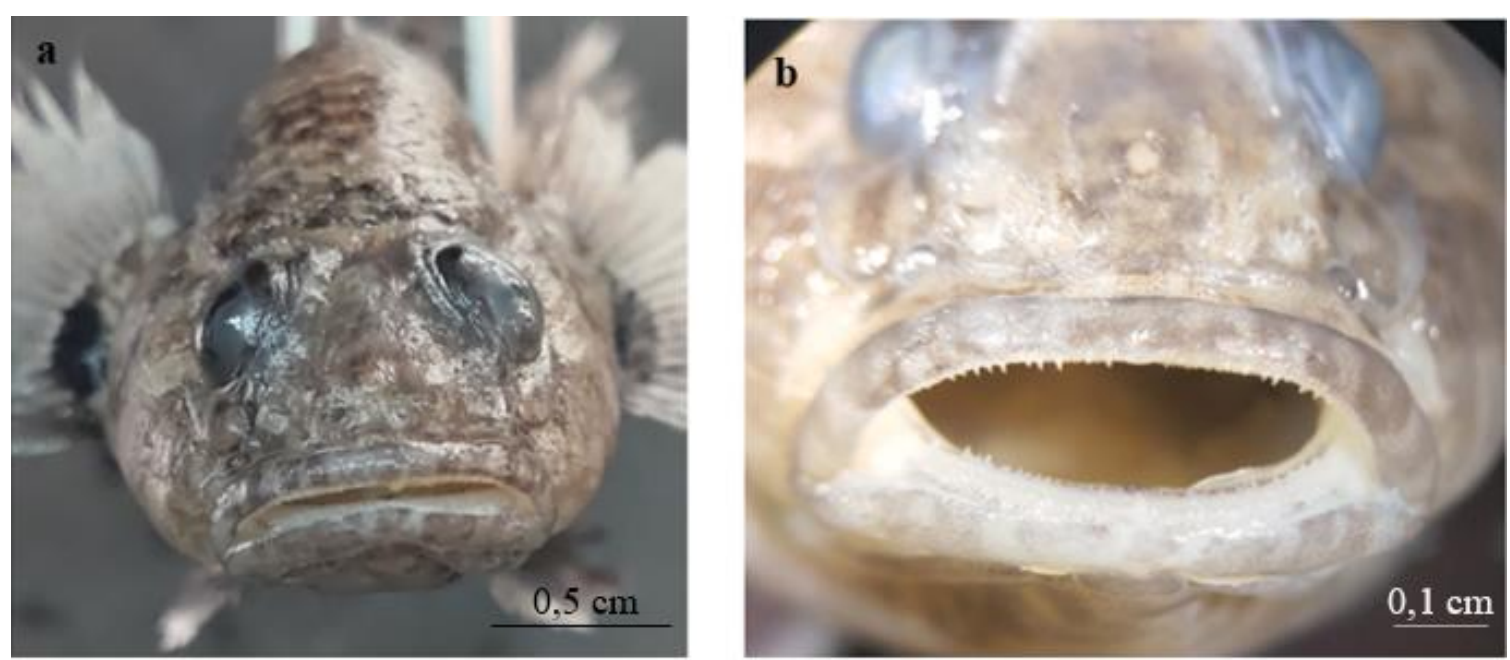

Hình 2. Miệng (a) và răng (b) của cá bống lưng cao Butis koilomatodon.

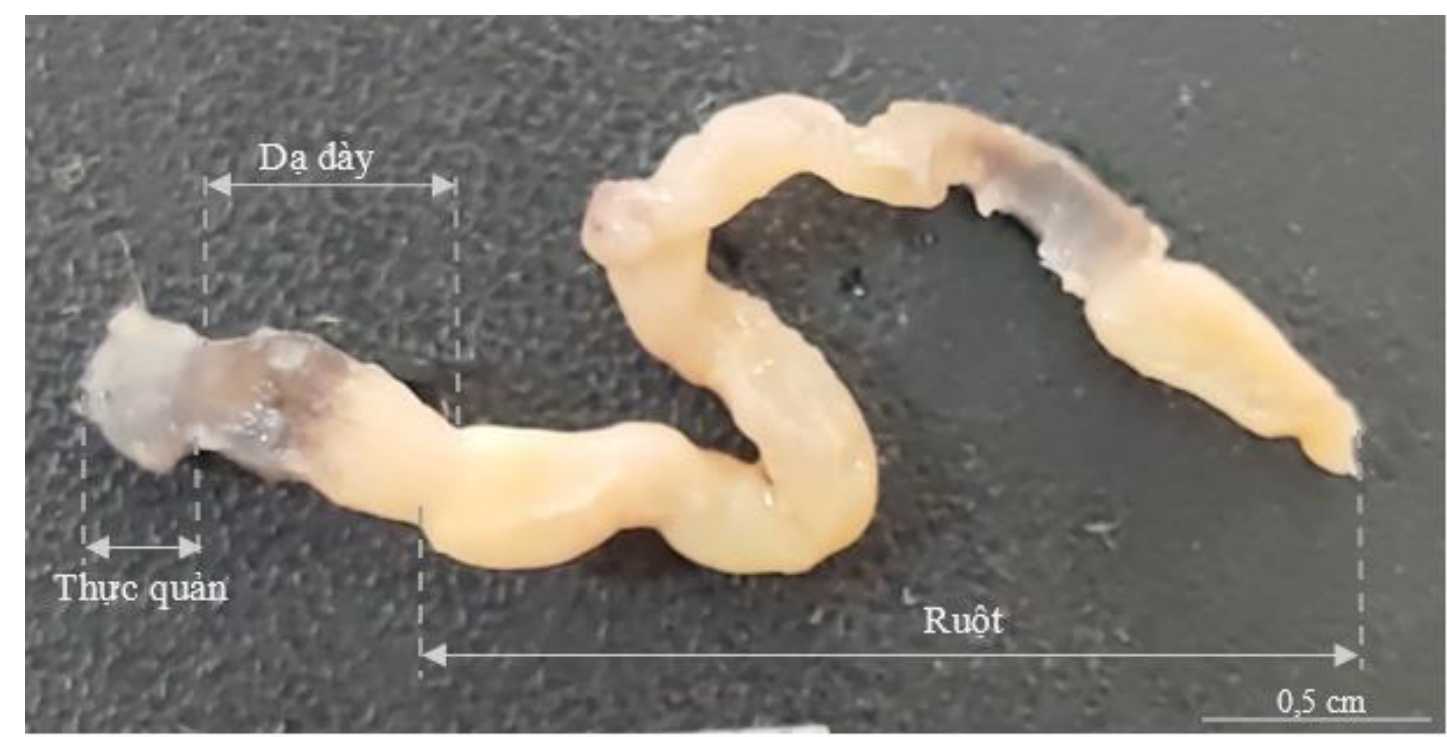

Hình 3. Ống tiêu hóa của cá bống lưng cao Butis koilomatodon.

\subsection{Hệ số béo Clark của cá bống lung cao Butis koilomatodon}

Hệ số béo Clark ở cá cái là $1,19 \pm 0,02 \mathrm{SE}$ (ttest, $\mathrm{n}=336, p>0,05)$, ở cá đực là 1,20 $10,02 \mathrm{SE}$ (t-test, $\mathrm{n}=891, p>0,05)$. Kết quả cho thấy ở cá đực hệ số béo tương đương cá cái, nhu cầu về dinh dưỡng của cá đực ngang bằng cá cái và không có ý nghĩa thống kê. Kết quả nghiên cứu này không chỉ gặp ở loài cá bống lưng cao Butis koilomatodon mà có một số loài khác cũng có hệ số béo Clark giữa cá đực và cá cái tương đương nhau (không có ý nghĩa thống kê) như Stigmatogobius pleurostigma [12], Periophthalmodon schlosseri [13], Eleotris melanosoma [10].

Hệ số béo Clark thay đổi theo kích thước của cá, với nhóm 1 (cá có kích thước nhỏ hơn chiều dài thành thục đầu tiên) có hệ số béo Clark là $1,30 \pm 0,06 \mathrm{SE}$ (t-test, $\mathrm{n}=232, p<0,05)$, cao hơn nhóm 2 (cá có kích thước lớn hơn chiều dài thành thục đầu tiên) có hệ số béo Clark là $1,17 \pm 0,01$ $\mathrm{SE}$ (t-test, $\mathrm{n}=995, p<0,05)$ (Hình 4). 


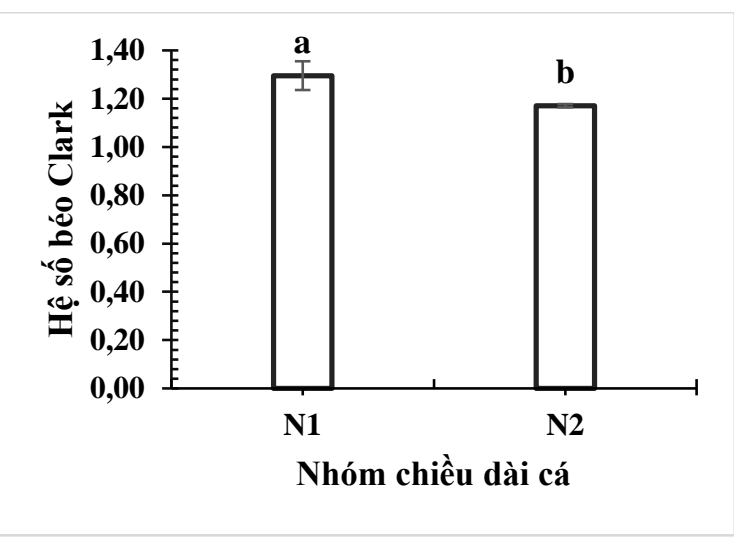

Hình 4. Sự biến động của hệ số béo Clark của cá bống lưng cao Butis koilomatodon theo nhóm chiều dài (N1: TL<chiều dài thành thục đầu tiên; N2: TL>chiều dài thành thục đầu tiên; Đường đứng: sai số chuẩn; $\mathrm{a}$ và $\mathrm{b}$ thể hiện sự khác biệt có ý nghĩa về mặt thống kê).

Sự khác nhau về hệ số béo Clark giữa 2 nhóm chiều dài có ý nghĩa về mặt thống kê và thấy được khả năng tích lũy năng lượng trong quá trình bắt mồi của nhóm cá chưa thành thục lớn hơn nhóm cá thành thục. Kết quả này khác với loài Periophthalmodon schlosseri [13] được nghiên cứu tại khu vực Trần Đề, Sóc Trăng có hệ số béo Clark tương đương nhau giữa cá chưa thành thục $(0,891 \pm 0,004)$ và nhóm cá thành thục $(0,869 \pm 0,008)$, với $p>0,05$.

Hệ số béo Clark của cá bống lưng cao Butis koilomatodon trong mùa khô là $1,20 \pm 0,01 \mathrm{SE}$ $(\mathrm{n}=658$, $\mathrm{t}$-test, $\mathrm{t}=0,68, p>0,05)$ và mùa mưa là $1,19 \pm 0,02$ SE $(\mathrm{n}=569, \mathrm{t}$-test, $\mathrm{t}=0,66, p>0,05)$ cho thây hệ số béo Clark không có sự khác nhau giữa hai mùa. Như vậy, lượng mưa không ảnh hưởng đến giá trị của hệ số béo Clark và loài này có sự thích nghi tốt khi môi trường thay đổi. Ở một số loài nhu: Parapocryptes serperaster [14], Stigmatogobius pleurostigma [12], Pseudapocryptes elongatus [15], Periophthalmodon schlosseri
[13] cũng có hệ số béo Clark không khác nhau giữa hai mùa khô và mùa mưa.

Trung bình hệ số béo của cá bống lưng cao Butis koilomatodon là 1,19 $\pm 0,01$ SE (1-way ANOVA, $n=1.227)$. Tại sáu điểm thu mẫu, hệ số béo Clark biến động từ 1,05 $\pm 0,01$ SE (Cù Lao Dung) đến 1,33 $\pm 0,03 \mathrm{SE}$ (Đầm Dơi). Chứng tỏ sự tích lũy năng lượng của loài cá này chịu tác động bởi yếu tố địa lý $(\mathrm{F}=10,37, p<0,05)$ (Hình 5). Trong thời gian nghiên cứu, giá trị hệ số béo Clark cũng có sự biến đổi giữa các tháng, đạt giá trị thấp nhất vào tháng $02 / 2020$ và cao nhất vào tháng $6 / 2019(\mathrm{~F}=8,84, p<0,05)$ (Hình 6).

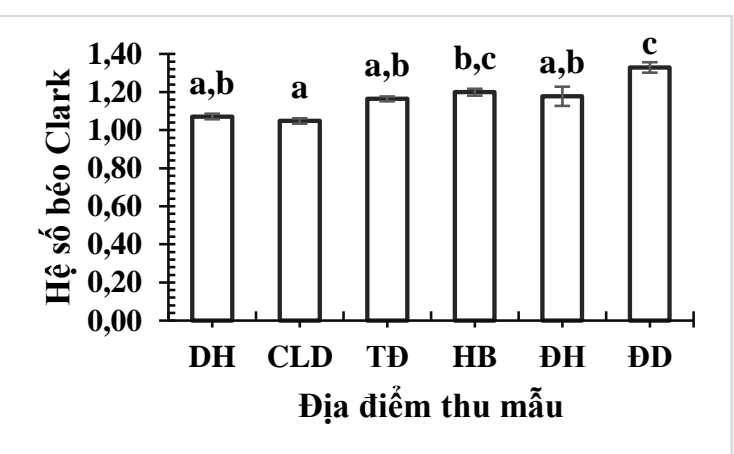

Hình 5. Sự biến động của hệ số béo Clark của cá bống lưng cao Butis koilomatodon tại các địa điểm thu mẫu (DH: Duyên Hải; CLD: Cù Lao Dung; TĐ: Trần Đề; ĐH: Đông Hải; Hòa Bình; ĐD: Đầm Dơi; Đường đứng: sai số chuẩn; $\mathrm{a}, \mathrm{b}$ và $\mathrm{c}$ thể hiện sự khác biệt có ý nghĩa về mặt thống kê).

Sự thay đổi giá trị hệ số béo Clark của loài này không chịu ảnh hưởng bởi sự tác động kết hợp của hai yếu tố giới tính $\mathrm{x}$ mùa (2-way ANOVA, $\mathrm{F}=2,47, p>0,05)($ Bảng 2 ) và giới tính $\times$ điểm thu mẫu $(\mathrm{F}=0,34, p>0,05)$ (Bảng 3$)$; nhưng chịu ảnh hưởng bởi sự tác động kết hợp của hai yếu tố chiều dài cá $\times$ mùa $(\mathrm{F}=32,31, p<$ $0,05)$ (Bảng 4), chiều dài cá $\times$ điểm thu mẫu $(\mathrm{F}=48,92, p<0,05)($ Bảng 5$)$ và mùa $\times$ địa điểm thu mẫu $(\mathrm{F}=11,76, p<0,05)$ (Bảng 6). 


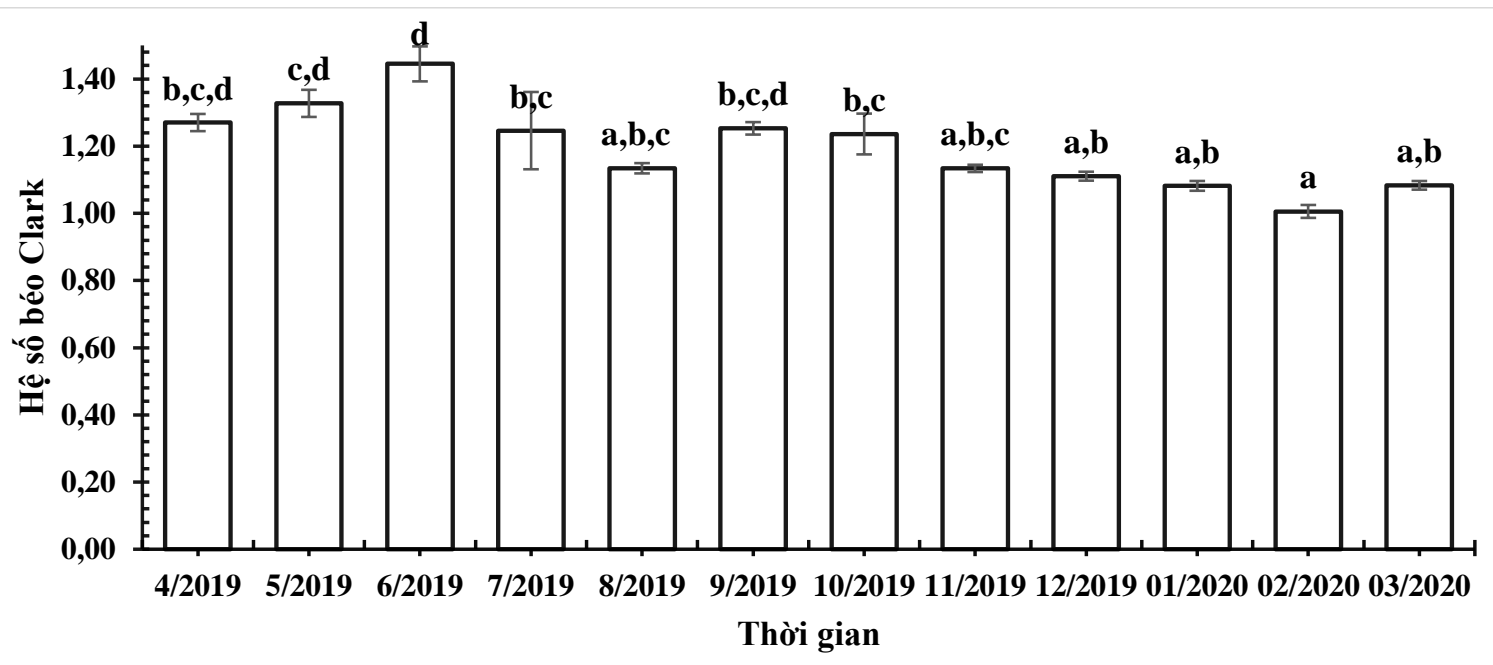

Hình 6. Sự biến động của hệ số béo Clark của cá bống lưng cao Butis koilomatodon trong 12 tháng thu mẫu (Đường đứng: sai số chuẩn; $\mathrm{a}, \mathrm{b}, \mathrm{c}$ và thể hiện sự khác biệt có ý nghĩa về mặt thống kê).

Bảng 2. Sự biến động của hệ số béo Clark của cá bống lưng cao Butis koilomatodon theo giới tính và mùa thu mẫu

\begin{tabular}{|c|c|c|}
\hline Giới tính & Mùa thu mẫu & Giá trị Clark (Trung bình \pm SE) \\
\hline \multirow{2}{*}{ Cá cái } & Mùa khô & $1,19 \pm 0,04$ \\
\cline { 2 - 3 } & Mùa mưa & $1,13 \pm 0,04$ \\
\hline \multirow{2}{*}{ Cá đực } & Mùa khô & $1,16 \pm 0,02$ \\
\cline { 2 - 3 } & Mùa mưa & $1,18 \pm 0,02$ \\
\hline
\end{tabular}

Bảng 3. Sự biến động của hệ số béo Clark của cá bống lưng cao Butis koilomatodon theo giới tính và địa điểm thu mẫu

\begin{tabular}{|c|l|c|}
\hline Giới tính & \multicolumn{1}{|c|}{ Địa điểm thu mẫu } & Giá trị Clark (Trung bình \pm SE) \\
\hline \multirow{5}{*}{ Cá cái } & Duyên Hải, Trà Vinh & $1,07 \pm 0,07$ \\
\cline { 2 - 3 } & Cù Lao Dung, Sóc Trăng & $1,08 \pm 0,09$ \\
\cline { 2 - 3 } & Trần Đề, Sóc Trăng & $1,21 \pm 0,10$ \\
\cline { 2 - 3 } & Hòa Bình, Bạc Liêu & $1,19 \pm 0,04$ \\
\cline { 2 - 3 } & Đông Hải, Bạc Liêu & $1,14 \pm 0,06$ \\
\cline { 2 - 3 } & Đầm Dơi, Cà Mau & $1,26 \pm 0,04$ \\
\hline \multirow{5}{*}{ Cá đực } & Duyên Hải, Trà Vinh & $1,10 \pm 0,04$ \\
\cline { 2 - 3 } & Cù Lao Dung, Sóc Trăng & $1,04 \pm 0,06$ \\
\cline { 2 - 3 } & Trần Đề, Sóc Trăng & $1,15 \pm 0,05$ \\
\cline { 2 - 3 } & Hòa Bình, Bạc Liêu & $1,20 \pm 0,03$ \\
\cline { 2 - 3 } & Đông Hải, Bạc Liêu & $1,19 \pm 0,03$ \\
\cline { 2 - 3 } & Đầm Dơi, Cà Mau & $1,33 \pm 0,03$ \\
\hline
\end{tabular}


Bảng 4. Sự biến động của hệ số béo Clark của cá bống lưng cao Butis koilomatodon theo nhóm chiều dài cá và mùa thu mẫu

\begin{tabular}{|c|c|c|}
\hline Nhóm chiều dài cá & Mùa thu mẫu & Giá trị Clark (Trung bình \pm SE) \\
\hline Nhóm 1 & Mùa khô & $2,12 \pm 0,06$ \\
\cline { 2 - 3 } (TL<chiều dài TTĐT) & Mùa mưa & $1,77 \pm 0,06$ \\
\hline Nhóm 2 & Mùa khô & $1,11 \pm 0,03$ \\
\cline { 2 - 3 } (TL>chiều dài TTĐT) & Mùa mưa & $1,20 \pm 0,03$ \\
\hline
\end{tabular}

Bảng 5. Sự biến động của hệ số béo Clark của cá bống lưng cao Butis koilomatodon theo nhóm chiều dài cá và địa điểm thu mẫu

\begin{tabular}{|c|l|c|}
\hline Nhóm chiều dài cá & \multicolumn{1}{|c|}{ Địa điểm thu mẫu } & Giá trị Clark (Trung bình \pm SE) \\
\hline \multirow{4}{*}{$\begin{array}{c}\text { Nhóm 1 } \\
\text { (TL<chiều dài TTĐT) }\end{array}$} & Duyên Hải, Trà Vinh & $0,87 \pm 0,08$ \\
\cline { 2 - 3 } & Cù Lao Dung, Sóc Trăng & $1,01 \pm 0,05$ \\
\cline { 2 - 3 } & Trần Đề, Sóc Trăng & $1,16 \pm 0,05$ \\
\cline { 2 - 3 } & Hòa Bình, Bạc Liêu & $4,05 \pm 0,27$ \\
\cline { 2 - 3 } & Đông Hải, Bạc Liêu & $2,88 \pm 0,15$ \\
\cline { 2 - 3 } & Đầm Dơi, Cà Mau & $1,67 \pm 0,05$ \\
\hline \multirow{4}{*}{$\begin{array}{c}\text { Nhóm 2 } \\
\text { (TL>chiều dài TTĐT) }\end{array}$} & Duyên Hải, Trà Vinh & $1,11 \pm 0,03$ \\
\cline { 2 - 3 } & Cù Lao Dung, Sóc Trăng & $1,17 \pm 0,10$ \\
\cline { 2 - 3 } & Trần Đề, Sóc Trăng & $1,11 \pm 0,05$ \\
\cline { 2 - 3 } & Hòa Bình, Bạc Liêu & $1,18 \pm 0,02$ \\
\cline { 2 - 3 } & Đông Hải, Bạc Liêu & $1,13 \pm 0,03$ \\
\cline { 2 - 3 } & Đầm Dơi, Cà Mau & $1,21 \pm 0,03$ \\
\hline
\end{tabular}

Bảng 6. Sự biến động của hệ số béo Clark của cá bống lưng cao Butis koilomatodon theo mùa và địa điểm thu mẫu

\begin{tabular}{|l|l|c|}
\hline Mùa thu mẫu & \multicolumn{1}{|c|}{ Địa điểm thu mẫu } & Giá trị Clark (Trung bình \pm SE) \\
\hline \multirow{4}{*}{ Mùa khồ } & Duyên Hải, Trà Vinh & $0,98 \pm 0,04$ \\
\cline { 2 - 3 } & Cù Lao Dung, Sóc Trăng & $1,03 \pm 0,07$ \\
\cline { 2 - 3 } & Trần Đề, Sóc Trăng & $1,13 \pm 0,06$ \\
\cline { 2 - 3 } & Hòa Bình, Bạc Liêu & $2,72 \pm 0,14$ \\
\cline { 2 - 3 } & Đông Hải, Bạc Liêu & $2,15 \pm 0,09$ \\
\cline { 2 - 3 } & Đầm Dơi, Cà Mau & $1,66 \pm 0,04$ \\
\hline \multirow{5}{*}{ Mùa mưa } & Duyên Hải, Trà Vinh & $1,00 \pm 0,07$ \\
\cline { 2 - 3 } & Cù Lao Dung, Sóc Trăng & $1,15 \pm 0,07$ \\
\cline { 2 - 3 } & Trần Đề, Sóc Trăng & $1,14 \pm 0,05$ \\
\cline { 2 - 3 } & Hòa Bình, Bạc Liêu & $2,52 \pm 0,14$ \\
\cline { 2 - 3 } & Đông Hải, Bạc Liêu & $1,85 \pm 0,08$ \\
\cline { 2 - 3 } & Đầm Dơi, Cà Mau & $1,22 \pm 0,04$ \\
\hline
\end{tabular}




\section{Kết luận}

Cá bống lưng cao Butis koilomatodon là một loài thuộc nhóm ăn động vật. Khả năng tích lũy năng lượng của loài này thể hiện qua hệ số béo Clark và không thay đổi theo mùa và giới tính nhưng thay đồi theo chiều dài của cá, địa điểm và thời gian thu mẫu. Kết quả nghiên cứu này đã bổ sung dẫn liệu khoa học về hình thái ống tiêu hóa và hệ số béo Clark qua đó làm cơ sở cho những nghiên cứu tiếp theo về nuôi nhân tạo loài cá này.

\section{Lời cảm ơn}

Chúng tôi xin chân thành cảm ơn Bộ Giáo dục và Đào tạo đã hỗ trợ về kinh phí cho đề tài này (B2019-TCT-02) và Nguyễn Hữu Đức Tôn, Nguyễn Thị Thúy Hiền và Trần Chí Cảnh đã hỗ trợ thu và phân tích mẫu.

\section{Tài liệu tham khảo}

[1] N.V. Hao, Freshwater fish of Viet Nam, vol 3, The Agricultural Publisher, Ha Noi, 2005.

[2] N.N. Thi, Fauna of Vietnam - Gobioidei, vol. 5, Sciences and Technics Publishing, Ha Noi, 2000.

[3] G.V. Nikolsky, Ecology of fishes, Academic Press, London, United Kingdom, 1963.

[4] F.N. Clark, The weight-length relationship of the California Sardine (Sardina cærulea) at San Pedro, Division of fish and game of California, California, 1928.

[5] T.D. Dinh, S. Koichi, N.T. Phuong, H.P. Hung, T.X. Loi, M.V. Hieu, U. Kenzo, Fishes of the Mekong Delta, Viet Nam, Can Tho University Publishing House, Can Tho, 2013.

[6] L.T.N. Thanh, Species composition and biological characteristics of some economic goby species in Bac Lieu and Soc Trang provinces, M.S. thesis, College of Aquaculture and Fisheries, Can Tho University, 2010.

[7] T.T. Kien, The composition of the goby species of Gobiidea and Eleotridae and some biological characteristics of the goby Glossogobius sparsipapillus Akihito and Meguro, 1976 in coastal area Thanh Phu district, Ben Tre province, M.S. thesis, College of Aquaculture and Fisheries, Can Tho University, 2013.
[8] V.T. Toan, T.D. Dinh, Study on nutritional characteristics of Oxyeleotris urophthalmus fish distributed along the Hau River, Science magazine of Can Tho University, vol. Thuy san, (2014) 192-197.

[9] D.M. Quang, N.T. Duy, D. Soc, Food and feeding habits of the broadhead sleeper Eleotris melanosoma from coastline in Soc Trang, in Proceeding of the 7 th National Scientific conference on Ecology and Biological Resources, Ha Noi, 2017, pp. 1873-1879.

[10] V.T. Toan, T.D. Dinh, D.T.H. Oanh, Study on nutritional characteristics of Broadhead sleeper (Eleotris melanosoma Bleeker, 1853) distribitung along Hau River, The $2^{\text {nd }}$ National Conference on Marine Biology and Sustainable Development, Science and Technics Publishing House, 2014, pp. 507-514.

[11] S.Z. Zulkifli, F. Mohamat-Yusuff, A. Ismail, N. Miyazaki, Food preference of the giant mudskipper Periophthalmodon schlosseri (Teleostei: Gobiidae), Knowledge and Management of Aquatic Ecosystems, 405 (2012) 07p01-07p10. https://doi.org/10.1051/kmae/2012 013.

[12] D.M. Quang, T.T.D. My, Digestive tract morphology, food and feeding habits of the goby Stigmatogobius pleurostigma (Bleeker, 1849) from the Coastline in Soc Trang, VNU Journal of Science: Natural Sciences and Technology, 34 (2018) 46-55. https://doi.org/10.25073/25881140/vnunst. 4740.

[13] T.T. Lam, H.D. Huy, D.M. Quang, Digestive tract morphology, food composition and feeding habits of the giant Mudskipper Periophthalmodon schlosseri (Pallas, 1770) from the Coastline in Tran De, Soc Trang, VNU Journal of Science: Natural Sciences and Technology, 35 (2019) 3038. https://doi.org/10.25073/2588-1140/vnunst. 4871.

[14] Q.M. Dinh, J.G. Qin, S. Dittmann, D.D. Tran, Seasonal variation of food and feeding in burrowing goby Parapocryptes serperaster (Gobiidae) at different body sizes, Ichthyological Research, 64 (2017) 179-189. https://doi.org/10. 1007/s10228-016-0553-4.

[15] D.D. Tran, Some aspects of biology and population dynamics of the goby Pseudapocryptes elongatus (Cuvier, 1816) in the Mekong Delta, $\mathrm{PhD}$ thesis, Universiti Malaysia Terengganu, 2008. 